\title{
Um fluxo proposto de condicionamento de dados pós-empilhamento para a interpretação sísmica
}

\author{
Wagner Moreira Lupinacci*, UFF \\ Allan Peixoto de Franco, UENF \\ Carlos André Martins de Assis, UNICAMP
}

Copyright 2016, SBGt - Sociedade Brasileira de Geofísica

Este texto foi preparado para a apresentação no VII Simpósio Brasileiro de Geofísica, Ouro Preto, 25 a 27 de outubro de 2016. Seu conteúdo foi revisado pelo Comitê Técnico do VII SimBGf, mas não necessariamente representa a opinião da SBGf ou de seus associados. É proibida a reprodução total ou parcial deste material para propósitos comerciais sem prévia autorização da SBGf.

\begin{abstract}
The interpretation of seismic data contributes to a better understanding of the depositional systems, helping to identify potential traps for oil accumulation. In order to improve the image of the seismic section after migration and stacking, this paper proposes a flow to condition the data for seismic interpretation. This flow consists of the following steps: estimation of the $Q$ factor, filtering in curvelet domain to remove noise and the application of a sparse deconvolution, which is able to compress the wavelet and recover the attenuation effects. The proposed flow was applied in a seismic section of the Pelotas Basin, and the result showed a considerable improvement in the identification and continuity of seismic events in more distant areas of the surface.
\end{abstract}

\section{Introdução}

A interpretação sísmica auxilia na construção de um modelo geológico conceitual de uma área em estudo. Frequentemente, os dados sísmicos migrados e empilhados apresentam uma quantidade significativa de ruídos, o que pode afetar a análise realizada pelo intérprete. É praticamente impossível a remoção de todo o ruído presente no dado, pois muitas vezes o ruído pode ser confundido com um sinal de baixa amplitude e ao retirá-lo podemos perder informações importantes para a interpretação sísmica. Existem diferentes técnicas de filtragem para remover parte deste ruído, como por exemplo: a utilização de filtros passa-banda, Radon e técnicas multiespectrais baseadas nas transformadas de Wavelet e Curvelet (Rangarajan et al., 2002; Gorszczyk et al., 2014; Franco e Moraes, 2015).

Além disso, a perda de resolução nos dados sísmicos causada pela atenuação dificulta o mapeamento de camadas delgadas e a interpretação de falhas, principalmente nas áreas mais distantes da superfície. Para corrigir os efeitos da atenuação, um filtro inverso $Q$ pode ser usado. Contudo, a utilização desta filtragem inversa tem um problema de instabilidade na compensação da amplitude. Então, é necessário ter certo cuidado ao utilizar este tipo de filtragem. Como exemplos de filtros inversos $Q$ estabilizados podemos ver (Wang, 2004, Braga e Moraes, 2013).

Outro problema é a presença do pulso sísmico que, em conjunto com a atenuação, é responsável pela limitação da banda de frequência do dado sísmico. Comumente, em um fluxo de processamento uma etapa de deconvolução é incluída com o objetivo de remover os efeitos do pulso sísmico e/ou reverberações. Na etapa de interpretação, aplicar a deconvolução esparsa em seções sísmicas empilhadas é uma opção interessante, pois pode fornecer um ganho adicional de resolução vertical (Oliveira e Lupinacci, 2013).

Neste trabalho, nos propomos um fluxo de précondicionamento de dados sísmicos pós-empilhamento para a interpretação sísmica, que é voltado para as camadas mais distantes da superfície. Este fluxo é compreendido por três etapas: estimativa do fator $Q$, aplicação de uma filtragem especial para remoção do ruído e utilização de uma deconvolução esparsa capaz de comprimir o pulso sísmico e compensar os efeitos de atenuação. Este fluxo foi aplicado em uma seção sísmica da Bacia de Pelotas em uma região caracterizada pela grande quantidade de hidratos de gás e zonas de gás livres. A presença destas camadas causa uma alta atenuação nos dados sísmicos, o que torna ainda mais interessante a aplicação deste fluxo nesta região.

\section{Metodologia}

O fluxo proposto de condicionamento para a interpretação sísmica de dados pós-empilhamento é divido em três etapas que são descritas nas seções seguintes.

\section{Estimativa do fator $\mathbf{Q}$}

Lupinacci e Oliveira (2015) avaliaram três alternativas para estimativa do fator Q. Estas alternativas são baseadas no decaimento exponencial do espectro de amplitude tempo-frequência do traço sísmico, que podem ser analisadas ao longo do tempo para um valor de frequência constante (método do decaimento da amplitude em função do tempo), ao longo de uma frequência para um tempo constante (método baseado na razão espectral) e ao longo da variável composto formada pelo produto do tempo com a frequência (método de Wang (Wang, 2004)). Eles concluíram que o último método é a estratégia mais robusta para a estimativa de fator de dados sísmicos empilhados. Então, o método de Wang foi o método selecionado para a estimativa do fator $Q$ traço a traço.

\section{Filtragem Curvelet}

A transformada curvelet, desenvolvida por Candès e Donoho (2000), é uma ferramenta que promove uma decomposição multiescalar e multidirecional de imagens. Segundo Candès e Demanet (2005), as curvelets apresentam superfícies suaves e um comportamento oscilatório na direção normal, semelhante à forma das ondas de propagação da sísmica, sendo assim essa 
ferramenta se torna uma ótima opção para decomposição desses tipos de dados, fornecendo um domínio altamente esparso que favorece a filtragem. A metodologia de aplicação da transformada curvelet utilizada nesse trabalho é abordada em: Fast Discrete Curvelet Transform (Candès et al., 2006).

A filtragem no domínio da curvelet se baseia na estimativa de um limite de corte para os coeficientes da transformada, no qual os menores valores tendem a conter somente ruído enquanto os coeficientes mais altos representam o sinal. A técnica de limite de corte utilizada para o fluxo proposto, denominada LMT (Local Multilevel Threshold), é proveniente do trabalho de Franco e Moraes (2015). Nesse trabalho, a estimativa do limite de corte para os coeficientes da transformada curvelet pode ser ajustada de acordo com a preferência do usuário, sendo realizada de forma diferencial para cada escala, orientação e localização.

\section{Deconvolução esparsa e correção da atenuação}

O método de deconvolução esparsa usado para obter a função refletividade do meio foi desenvolvido por Oliveira e Lupinacci (2013). Um diferencial desta implementação é a inclusão do tratamento dos efeitos de atenuação, por meio do fator $Q$, que pode ser entendida como uma forma de considerar o pulso sísmico não estacionário (Yilmaz, 2001). Para isto, a deconvolução é realizada no domínio da frequência, no qual a modelagem dos efeitos da atenuação é mais simples e intuitiva do que no domínio do tempo. Além disso, o processo é formulado como um problema inverso, composto por uma função objetiva a ser minimizada usando a norma L1 no resíduo dos dados e no modelo dos parâmetros. A utilização da norma L1 no resíduo dos dados impõe robustez ao processo de deconvolução, diminuindo a influência de ruídos aleatórios. $\mathrm{E}$ a aplicação desta norma nos parâmetros promove a esparsidade da solução. O termo de regularização é ponderado por um parâmetro responsável por controlar a esparsidade da solução. Um baixo valor deste parâmetro se traduz em um melhor ajuste dos dados, gerando assim uma solução não esparsa. E um alto valor do parâmetro de regularização gera uma solução concentrada em poucos impulsos perdendo informações importantes, como as reflexões de pequena amplitude. Atualmente este parâmetros é escolhido por meio de uma inspeção visual do intérprete.

Oliveira e Lupinacci (2013) mostraram que esta ferramenta é capaz de comprimir o pulso sísmico e recuperar parte dos efeitos da atenuação. É interessante observar que a compressão do pulso obtida no domínio do tempo equivale no domínio da frequência à recuperação da magnitude das altas frequências presentes nos dados.

\section{Resultados e Discussões}

O fluxo proposto para condicionar a interpretação sísmica foi aplicado em uma seção da Bacia de Pelotas (Figura 1). A seção empilhada foi obtida através de um simples fluxo de processamento que consistiu de correção do espalhamento geométrico, deconvolução adaptativa, análise de velocidade, correção dip-moveout, migração pre-stack (Stolt) e filtragem passa-banda. a)

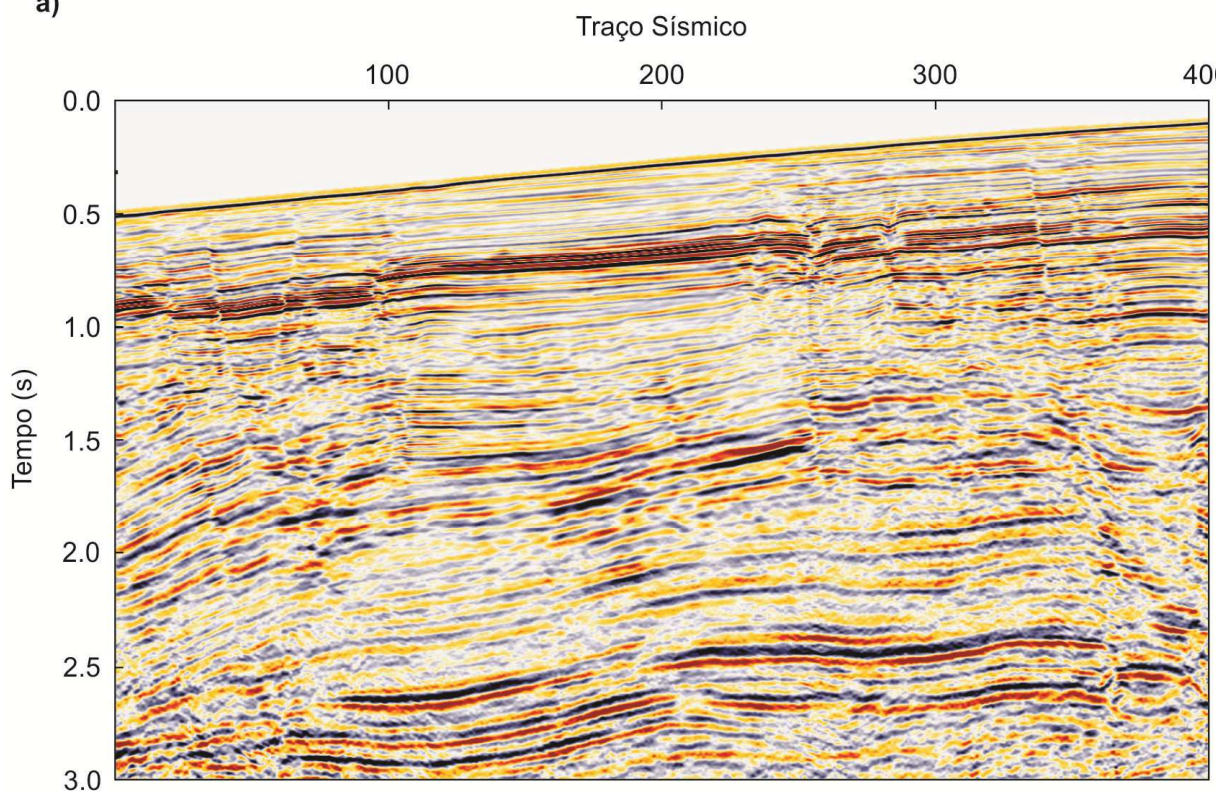

b) Frequencia $(\mathrm{Hz})$ $\begin{array}{llllll}400 & 0 & 25 & 50 & 75 & 100\end{array}$

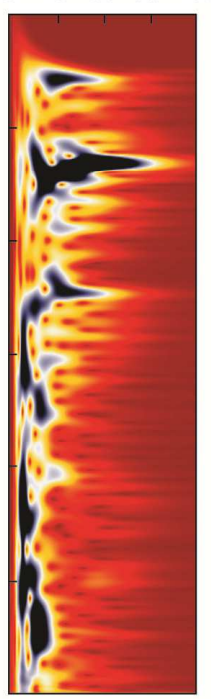

Figura 1 - (a) Seção sísmica da Bacia de Pelotas. (b) Espectro de amplitude variando com o tempo da seção sísmica.

No primeiro estágio realizamos a estimava do fator $Q$ traço a traço usando o método de Wang. Em cada traço, nós estimamos o fator $Q$ usando uma janela temporal de 0-2.0s e repetimos em uma janela temporal de 1.0-3.0s.
Em seguida, uma media móvel foi usada para suavizar lateralmente os valores encontrados. Os resultados da estimativa do fator $Q$ são mostrados na figura 2 (curva vermelha para a janela 0-2.0 e curva azul para a janela 
1.0-3.0s). Para construir uma matriz de fatores $Q$ variando com o tempo e lateralmente, nos usamos os valores da curva vermelha no intervalo de $0-1.0 \mathrm{~s}$, os valores da curva azul no intervalo de 2.0-3.0s e aplicamos uma interpolação linear baseada nos valores de 1.0s a 2.0s. Esta foi a matriz Q usada como parâmetro de entrada na deconvolução esparsa. Os valores do fator $Q$ foram maiores na segunda janela temporal. Isto ocorre porque normalmente as camadas mais distantes da superfície são mais compactadas, o que provoca uma diminuição dos efeitos da atenuação na propagação das ondas sísmicas. Outro fato que também contribuiu para os valores do fator $Q$ na primeira janela serem menores é a existência de uma grande acumulação de hidratos de gás e zonas de gás livre próximas ao fundo oceânico, no qual contribui para a alta atenuação das ondas sísmicas.

Através da filtragem LMT nós obtivemos uma seção sísmica com menor quantidade de ruído (Figura 3). Os ganhos que uma imagem menos ruidosa proporcionam à interpretação são ilustrados em uma análise dos primeiros 100 traços, principalmente em regiões nas profundidades maiores. Nessa região a continuidade e identificação dos refletores se sobressaem na seção

a)

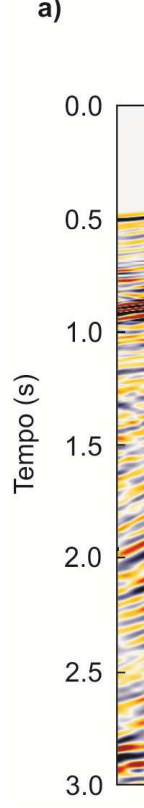

Traço Sísmico

100 200 300 análise de cada painel de escala e orientação proveniente da transformada curvelet visando retirar manualmente os painéis que apresentavam uma lata quantidade de ruído. Essa alternativa é capaz de auxiliar, principalmente, na remoção de ruído coerente. Nesse trabalho, nós procuramos utilizar uma parametrização mais suave e conservadora visando preservar as amplitudes e as falhas da seção. Embora as falhas ficaram mais suaves ainda são fácies de identificar.

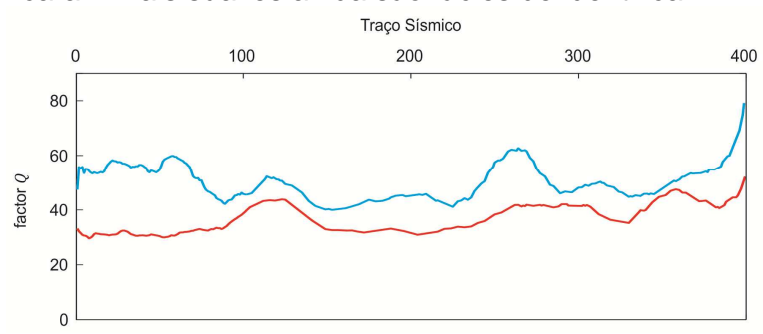

Figura 2 - Valores do fator $Q$ estimados da seção sísmica da Bacia de Pelotas para uma janela de 0-2.0s (curva vermelha) e uma janela de 1.0-3.0s (curva azul).

b) Frequencia $(\mathrm{Hz})$ $\begin{array}{llllll}400 & 0 & 25 & 50 & 75 & 100\end{array}$

Figura 3 - (a) Seção sísmica após a aplicação da filtragem curvelet. (b) Espectro de amplitude variando com o tempo da seção sísmica após a filtragem curvelet.

Após a aplicação da filtragem, nós realizamos alguns testes com a deconvolução esparsa para escolher o parâmetro de regularização capaz de obter uma solução esparsa e ao mesmo tempo preservar o máximo de sinal possível. Com este parâmetro selecionado, a deconvolução esparsa foi usada tendo como entrada o dado sísmico, a matriz de fator $Q$ e o parâmetro de regularização. Em um primeiro momento a utilização da matriz original do fator $Q$ compensou as perdas de amplitudes além do esperado. A fim de se contornar o ocorrido nós adicionamos um valor constante à matriz visando minimizar a compensação exagerada obtida.
O resultado final do fluxo proposto, após a deconvolução esparsa, é mostrado na Figura 4. Com a utilização deste fluxo, podemos notar uma melhoria considerável na continuidade dos eventos sísmico e um ganho expressivo de resolução (o que pode ser observado através do espectrograma mostrado na Figura 4b) nas camadas mais distantes da superfície. Dessa forma é possível distinguir refletores que antes se confundiam em um só. Além disso, as reflexões entre camadas de pequeno contraste também foram preservadas. 


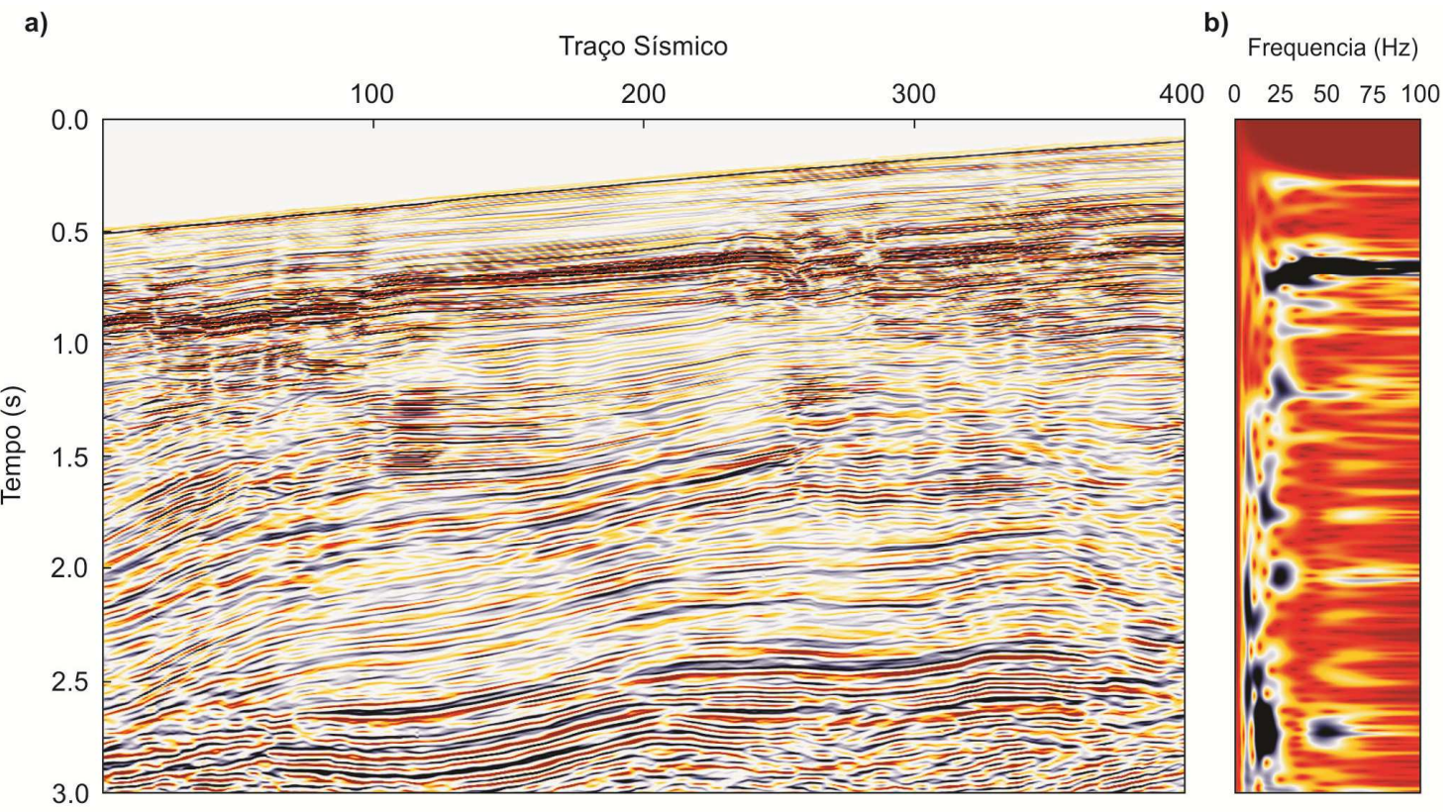

Figura 4 - (a) Seção sísmica após a aplicação do fluxo proposto. (b) Espectro de amplitude variante com o tempo da seção sísmica após o fluxo proposto.

\section{Conclusões}

O resultado do fluxo proposto aplicado na seção sísmica da Bacia de Pelotas mostrou ser capaz de remover parte do ruído, comprimir o pulso sísmico e compensar os efeitos da atenuação. Portanto, o fluxo proposto pode ser uma maneira adicional e eficaz a ser considerada na etapa de interpretação sísmica de eventos distantes da superfície.

\section{Agradecimentos}

Os autores agradecem à Agência Nacional de Petróleo (ANP) pelo fornecimento dos dados sísmicos utilizados nesta pesquisa.

\section{Referências}

Braga, I. L. S., Moraes, F. S., 2013. High-resolution gathers by inverse $Q$ filtering in the wavelet domain. Geophysics 78, V53-V61.

Candés, E. J., Demanet, L., 2005. The curvelet representation of wave propagators is optimally sparse. Communications on Pure and Applied Mathematics, v. 58 (11): 1472 - 1528.

Candès, E. J., Demanet, L., Donoho, D., Ying, L., 2006. Fast Discrete Curvelet Transforms. Society for Industrial and Applied Mathematics, Multiscale Model. Simul. vol. 5(3): 861-899.

Candés, E. J., Donoho, D. L., 2000. Curvelets - A surprisingly effective nonadaptive representation for objects with edges. Technical Report, Department of Statistics, Stanford University, California, USA.
Franco, A. P., Moraes, F. S., 2015. Local Multilevel Threshold in the curvelet domain. $14^{\text {th }}$ International Congress of the Brazilian Geophysical Society.

Gorszczyk, A.; Adamczyk, A.; Malinowski, M., 2014. Application of curvelet denoising to $2 \mathrm{D}$ and $3 \mathrm{D}$ seismic data - Practical Considerations. Journal of Applied Geophysics vol. 105: 78-94.

Lupinacci, W. M., Oliveira, S. A. M., 2015. Q factor estimation from the amplitude spectrum of the timefrequency transform of stacked reflection seismic data. Journal of Applied Geophysics vol. 114: 202-209.

Oliveira, S. A. M. and Lupinacci, W. M., 2013. L1 norm inversion method for deconvolution in attenuating media. Geophysical Prospecting vol. 61: 771-777.

Rangarajan, R.; Venkataramanan, R.; Shah, S., 2002. Image Denoising Using Wavelets. [S.I]. Wavelets \& Time Frequency.

Wang, Y., 2004. $Q$ analysis on reflection seismic data. Geophysical Research Letters, vol. 31: L17606.

Yilmaz, O., 2001. Seismic Data Analysis: Processing, Inversion and Interpretation of Seismic Data. 2nd Edition Hardcover. 